\title{
A Story of Science: del cómo el análisis en las narrativas y los canales de comunicación emulsionan a las generaciones en la divulgación científica
}

\author{
Germán Emiliano Calderón Carmona ${ }^{1}$
}

\begin{abstract}
Resumen
En la actualidad se habla de procesos de adaptación mucho más exigentes en el fenómeno comunicativo del campo de la divulgación científica. Esto, a partir de las nuevas tendencias de inmediatez, simplificación y distribución de la información. Hoy se requieren de análisis mucho más rigurosos sobre la eficacia de las herramientas y las formas utilizadas en la comunicación especializada. Existe un llamado de urgencia en la divulgación científica, cuyo principio requiere la creación de una vinculación propositiva que genere un ejercicio comunicativo flexible en la ciencia, contribuyendo de forma efectiva, plural y positiva a la comunidad científica y general.

Abstract

Nowadays there is a requirement of adaptive procedures in the communication phenomenon in the field of Scientific Dissemination. This, based on the new trends of immediacy, simplification and distribution of information. Today, a much more rigorous analysis of the effectiveness of tools and forms used in specialized communication is required. There is an urgent call in the Scientific Dissemination field, whose principle requires the creation of a proactive linkage that generates a flexible communicative exercise in science, contributing to the scientific and general community in an effective, plural and positive way.

Palabras Clave

Divulgación científica, Canales complementarios, Nativos digitales, Comunicación científica y transmedia. Keywords

Science dissemination, Complementary Channels, Digital natives, Science communication and transmedia.

*Tercer lugar del concurso del Trabajos Recepcionales en Comunicación CONEICC 2019. Universidad Anáhuac Campus Puebla. Asesores de tesis: Mtro. Ricardo Hernández Briones, Mtro. Gregorio Moisés Romero Olvera, Dr. Carlos Giordano Sánchez Verín y Dr. Juan José Gómez Díaz.

${ }^{1}$ Autor para correspondencia: gcalderoncarmona@gmail.com
\end{abstract}

\section{Introducción}

En la actualidad, la creación, promoción y difusión de la divulgación científica, son algunos de los tantos pilares del desarrollo humano de las naciones. La ciencia y la investigación son dos fuerzas importantes de cambio en la realidad presente, capaces de afectar el ritmo y la organización de la sociedad. Consecuencias positivas de ello se engloban en diferentes sectores, dando como resultados la
Educación STEM ${ }^{1}$, el impulso a la infraestructura ingenieril, los programas educativos especializados en ciencia, los sistemas nacionales de investigación, la inversión nacional y extranjera en áreas científicas y más.

La publicación de escritos e investigaciones en revistas especializadas, anuarios de investigación, así como repositorios digitales, forman parte de una de las áreas de

\footnotetext{
${ }^{1}$ STEM responde al acrónimo en inglés: Science, Technology, Engineering and Mathematics (Ciencia, Tecnología, Ingeniería y Matemáticas). El término STEM fue creado en los años noventa por la National Science Foundation (NSF) (Sanders, 2009).
} 
mayor presencia en el campo de la divulgación científica. Sin embargo, la realización de esta faena se enfrenta a diferentes retos y desafíos.

En primera instancia, se puede hablar de los principios básicos para la realización de la divulgación científica: los aspectos económicos y políticos. A nivel nacional, el gobierno de México es responsable de fomentar, a través de políticas públicas, la realización y el crecimiento de la investigación en las instituciones de educación, ciencia y tecnología. Mediante sus órganos centralizados (v.gr. la Secretaría de Educación, Ciencia, Tecnología e Innovación [SECTEI]) o los descentralizados (v.gr. Consejo Nacional de Ciencia y Tecnología [CONACYT]) se contemplan acciones para la vinculación de la ciencia con la sociedad.

No obstante, dichos actores y planes han imposibilitado la generación de contenido científico en México. La reducción en los porcentajes del Producto Interno Bruto (PIB) Anual a los Programas y planes en apoyo a la ciencia se ven reflejados en fenómenos desde las fugas de cerebros ${ }^{2}$ hasta una disminución en el carácter cuantitativo y cualitativo de las investigaciones científicas. Tan sólo el $70 \%$ de las publicaciones anuales son producidas por instituciones y universidades públicas que reciben la mayor parte del presupuesto público para las ciencias. Mientras que el $30 \%$ de las publicaciones anuales, realizadas por instituciones de carácter privado, carecen en un gran aspecto de apoyo financiero para la investigación y la promoción de las ciencias (Ibarra \& Villagrán, 2013).

Por otra parte, la consolidación de los ejercicios y esfuerzos de divulgación científica están basados en la construcción correcta, eficiente y eficaz de los modelos de comunicación. Este eje no sólo incluye el uso de aspectos básicos en gramática, ortografía o corrección de estilo. Sino que también involucra el uso de los diferentes canales de comunicación (tradicionales y digitales) donde las investigaciones puedan acceder a nuevas formas narrativas. En este último punto, se puede, de igual manera, agregar las capacidades de adaptación y elasticidad que deben tener los textos científicos tomando en cuenta diferentes metas:

\footnotetext{
${ }^{2}$ La fuga de cerebros es un término acuñado por la prensa británica en los años 60. Este concepto define a los profesionales que han desarrollado una carrera universitaria en su país y emigran a otros sitios para continuar con su educación, desarrollar investigaciones o encontrar mejores oportunidades de trabajo (UNAM, 2013).
}

audiencia potencial y real, impacto académico y social, así como, capacidad de extensión/continuidad de la investigación por otros investigadores.

\section{La crisis de la divulgación científica en la comunicación}

Estamos ante una situación crítica donde el paradigma comunicativo de la divulgación científica requiere de una nueva propuesta aplicada para el fomento del conocimiento en México. Ya lo decía Manuel Calvo (2005), hace más de una década: "tal vez en ninguna otra época la ciencia haya necesitado como ahora de la mano amiga de la comunicación, no tanto para aplaudirla como creadora de hechos memorables sino como para explicarla de la manera más amplia” (p. 1).

No sólo las políticas públicas o las vastas extensiones comunicativas modernas son parte de los retos actuales. También la falta de retroalimentación en los procesos metodológicos utilizados actualmente por los divulgadores actuales. La creación de investigaciones para el crecimiento del acervo literario cuyo destino final es la misma academia y que forman parte de procesos burocráticos, se integran a una tradición que podría ser tachada de poco propositiva y desinteresada por el desarrollo de la comunidad científica potencial.

Los investigadores actuales, en su mayoría, conservan el canon y la estética rígida en las publicaciones científicas. Las publicaciones con exceso de tecnicismos, títulos poco atractivos para un público general, un acceso al acervo literario restringido a la comunidad que no es parte de la academia, textos extensos y repetitivos, entre otros, pueden ser ejemplos de una manera ortodoxa que puede llegar a carecer de elasticidad y que no toma como fin poner la ciencia al alcance de la población (Calvo, 2005).

¿Qué es lo que requiere entonces la comunidad científica cuando los llamados de atención y urgencia se han presentado desde el siglo pasado en textos claves como Experiencias de la divulgación de tecnología y ciencia en México (1985) del antiguo Consejo Nacional del Sistema Nacional de Educación Técnica (COSNET); La Declaración de la Ciencia sobre la ciencia y el uso del saber científico (1999) de la UNESCO o en diferentes textos de los últimos quince años de los teóricos 
en la divulgación científica como Luis Estrada $(1992,2014)$ o Aquiles Negrete (2008)?

Es aquí cuando el papel de la comunidad comunicóloga aparece como un elemento clave para el futuro en la crisis de la divulgación científica actual. La ventana de oportunidad en el estudio y la propuesta de soluciones para esta problemática es amplia y llena de elementos de investigación. El comunicólogo es ahora uno de los agentes de cambio requeridos para asistir a los divulgadores a través de sus conocimientos en medios, cultura organizacional, diseño editorial, comunicación visual y más. A partir de una revisión en los programas estudiantiles de las ciencias de comunicación, los comunicólogos actuales, están preparados para poder aplicar sus conocimientos generales y especializados en temas como este.

\section{Nuevos retos: los nativos digitales}

Dentro de la misma investigación de tipo explicativo realizada en el periodo 2017-2018 en el Estado de Puebla (México), se identificó un elemento clave que fue parte importante del desarrollo de dicho estudio. El llamado "nativo digital", como lo establece Prensky (2001), es una denotación social para aquellas personas nacidas entre el boom de la era digital (el inicio del internet, la telefonía celular y/o las nuevas tendencias digitales). Los nativos digitales crecieron con el inicio de las redes sociales y con una capacidad casi innata para el fácil manejo de las computadoras. Por ello, desde temprana edad, dicho público se vio sobre-impactado por una nueva forma de consumo mediático e informativo.

Los nativos digitales se diferencian de los migrantes digitales, aquellos nacidos antes del auge de la era digital (Prensky, 2001), no sólo en la manera en que procesan la información, sino también en el tipo de información que están acostumbrados a consumir. Dicho público, consume mayor contenido multimedia visual que textual. Por ello, los medios tradicionales para la distribución de información como lo son los libros, la radio o la televisión, se han visto en la necesidad de adaptar sus formatos a extensiones menores de texto o tiempo de reproducción así como el enfrentamiento a un fenómeno donde el usuario final se convierte en el creador de contenido principal (prosumidores).
Para los modelos educativos, mercadológicos, informativos $\mathrm{y}$ de entretenimiento, el nativo digital ha representado conflictos debido a su acelerada metamorfosis que ha sido, en parte, generada por la constante transformación de los medios y la tecnología. La creación de sistemas para establecer leyes en cuanto al funcionamiento en el consumo y la producción de información, no han podido en su mayoría ajustarse para lograr los diagnósticos correctos y propuestas de niveles de acción.

La rama de la sociología ha evidenciado que el nativo digital varía entre subgeneraciones; es decir, el nativo digital nacido en los mediados de los noventa, encontrará una diferencia casi abismal conforme a las conductas y formas de coexistencia de un nativo digital nacido en los años 2000. Este fenómeno puso en jaque las investigaciones sobre el desarrollo de la sociedad, como lo fue Generations (1991) de Howe y Strauss, que buscaba anticipar las conductas y los desafíos a los que se enfrentarían las generaciones de los años noventa y los del nuevo milenio.

Así como la sociología, a través de Howe y Strauss (1991), por ejemplo, no pudo advenir el acelerado cambio generacional, la divulgación científica, en gran escala, pasó por desapercibidas las nuevas herramientas que el veloz desarrollo tecnológico comenzó a ofrecer. También, las nuevas narrativas que comenzaron a gestarse en la última década y que sin lugar a duda serían necesarias para poder acceder a los nuevos públicos.

\section{Una adaptabilidad intermitente}

La divulgación científica es una tarea que se ha adaptado a las necesidades de la sociedad desde hace varios siglos. Con resultados como la creación de comunidades y sociedades como la Royal Society fundada en 1662, que tuvo miembros destacados como Newton, Francis Bacon, y/o Darwin (Royal Society Org, s.f.) hasta la Academie Des Sciences de París fundada en 1666 (Hatch, s.f.), para llegar finalmente a las ferias internacionales de ciencia juveniles que involucran a cientos de países en eventos a escalas mayores.

De la misma forma, podemos ejemplificar la capacidad de respuesta que se ha tenido dentro del país. Con resultados en la creación de infraestructura tecnológica para las nuevas 
asociaciones científicas, la propuesta continua de centros de investigación e incubadoras de las universidades, la promoción de eventos culturales de corte científico y hasta museos especializados para la promoción de las ciencias.

Existe evidencia de que la divulgación científica y la sociedad en general ya se han adaptado en algunas ocasiones a las exigencias de la sociedad para la vinculación del haber y saber científico. Consecuencias positivas de ello son los shows televisivos como Cosmos: Un viaje personal en 1980 presentado por el divulgador Carl Sagan y su secuela Cosmos: A Spacetime Odyssey (2014) presentada por Neil deGrasse Tyson, ambos dirigidos a un público maduro; Bill Nye the Science Guy (1993), El Mundo de Beakman (1997) o The Cat in the Hat Knows a Lot About That! (2010), dirigidos hacia las generaciones más jóvenes.

De igual manera, no sería la primera vez que una adaptación en el formato de comunicación ocurre dentro de las publicaciones científicas textuales. Tan sólo propuestas como What If?: Serious Scientific Answers to Absurd Hypothetical Questions (2014) de Randall Munroe, Astrophysics for People in a Hurry (2017) de Neil DeGrasse Tyson y hasta el bestseller A Brief History of Time (1998) de Stephen Hawking son ejemplos de cómo una propuesta de diseño editorial y de extensión de contenido aplicado a ciertos públicos fuera de la academia pueden ayudar a que la divulgación científica opte por nuevos caminos en la comunicación.

Por lo tanto, no existiría un razonamiento lógico que justifique la incapacidad de adaptabilidad de la divulgación científica actualmente, ante el crecimiento exponencial del formato de consumo de los nativos digitales. $\mathrm{O}$ bien, una razón por la que ante las cambiantes formas narrativas requeridas para la comunicación, no se puedan ofrecer nuevos formatos en publicaciones que sean atractivas y funcionales a las necesidades de estos públicos difíciles de acceder.

\section{Canales complementarios y narrativas transmedia}

Gracias a las diferentes investigaciones de teóricos como Santos García (2012), Thompson (2012) o Álvarez (Álvarez en Cedaro, 2007), podemos definir a un canal complementario en la comunicación como: Aquel canal cuya función principal es apoyar al canal principal que está siendo utilizado para compartir y transmitir información. El canal complementario auxilia y se diferencia del canal principal por sus capacidades de alcance en la exploración de nuevos simbolismos, lenguajes y distribución de dicha información que apoyen a la comprensión de la primera emisión de información. El fin principal, no es superar al canal original, sino convertirse en un puente auxiliar en el ejercicio de los sistemas de comunicación físicos así como digitales existentes y/o por existir.

El uso de un canal complementario reside en los esfuerzos comunicativos donde la información codifica nueva e inteligentemente para que pueda ser mejor comprendida por el público meta original o un segundo público potencial. Dentro de las nuevas tendencias de la comunicación y su relación con los medios, no sólo se busca la creación de nuevos canales sino también se vela por nuevas estrategias que hagan converger a los medios existentes en pro de mejores prácticas comunicativas.

El concepto de narrativas transmedia fue introducido por el investigador estadounidense Henry Jenkins en el año 2003 (Scolari, 2014). Las narrativas transmedias tienen dos rasgos importantes:

1. Son relatos funcionales que se cuentan a través de diferentes y múltiples medios o plataformas.

2. Las narrativas transmedia no se limitan solamente a ser consumidas por el usuario final, sino que también, este mismo se embarca en la tarea de ampliar este universo narrativo.

Como ejemplo del primer rasgo, podemos dar lugar a que la narrativa comienza, en formato de tira cómica, para después trasladarse a un formato televisivo y finalmente llegar a convertirse en un largometraje. Esto involucra, obviamente, técnicas de narración y estéticas diferentes. No es lo mismo, una historia contada en 20 minutos para un formato de TV que un formato de 90 minutos para cine; cada uno requiere de un lenguaje especializado en cuanto a fotografía, técnicas de grabación, ensamblaje y edición final, entre otros.

La transmedia no influye en que la historia cambie de canal comunicativo per se. Sino que en cada formato (v. gr. tira cómica, televisión y cine) la historia se extienda a través 
de la anexión de nuevos personajes, escenografías, scripts, actores y más.

El universo narrativo de sagas de la cultura popular como lo son Harry Potter, Star Wars o la marca de juguetes para niños $L E G O$ son ejemplos de formatos transmediáticos exitosos. Harry Potter es uno de los ejemplos y casos de estudio transmediáticos más importantes de los últimos años.

La novela inglesa publicada por Joanne Rowling, obtuvo su primera publicación 1997 por la Casa Editorial Bloomsbury (Inglaterra), con tan sólo 500 ejemplares. Tras el éxito en ventas, el universo literario de Harry Potter no sólo creció en más secuelas del primer libro, sino que los personajes y las historias fueron llevadas a nuevos medios. Así, Harry Potter fue adaptado y expandido narrativamente en cine, juguetes, libros, podcasts, videojuegos, una obra de teatro y hasta una red social llamada Pottermore donde los usuarios tienen la capacidad de aumentar el universo narrativo, no de forma oficial, sino en un canon alterno al original (Jenkins, 2011).

El uso de canales complementarios y de narrativas transmedia requiere de experimentación, sistematización, diagnósticos constantes de eficacia y de pruebas de calidad en cuanto a la eficiencia de los canales utilizados para la extensión de la comunicación. Es así, que las estrategias, posiblemente, requieran para un exitoso proceso de exploración de antecedentes donde los canales complementarios y la transmedia hayan sido utilizados. De igual manera requiere de equipos integrales con comunicólogos especializados y varias disciplinas que coadyuven en un sólo fin: el de la comunicación exitosa y expandible.

\section{Hacia una propuesta de re-adaptación en la divulgación científica}

Dentro de la divulgación científica y los trabajos de investigación publicados, el proceso de transmedia precisa de importantes tareas claves. Es imperante que para lograr una readaptación, las publicaciones científicas originales se sometan a revisiones pertinentes de forma y fondo en cuanto a su narrativa. Dentro de ello, podemos tomar en cuenta factores como la extensión en el número de palabras, la presentación visual, el uso (y adecuación) de los tecnicismos, la sobreexplotación de recursos textuales como las notas al pie de página que abarcan una gran parte de la hoja y la falta de medios visuales como imágenes en los textos, así como otros.

Uno de los ejercicios más exitosos de dicha revisión en cuanto al contenido editorial de un documento científico es el del bestseller de Stephen Hawking Historia del Tiempo (1988), donde el divulgador confesó en el prólogo del libro que la reducción de ventas tendría una relación directa con cada ecuación que incluyera en su libro. Finalmente, es comprensible la misión de Hawkins (1998): la de compartir la mayor información posible sobre sus descubrimientos y propuestas. Sin embargo la decisión de Hawkins (1998) de reducir la cantidad de ecuaciones de sus investigaciones va más allá de la búsqueda de ventas de ejemplares y puede suponer un interés por el que el auditorio receptor se acerque y no rechace un libro de física cuántica y de partículas, los cuales son conocidos por su gran cantidad de datos y análisis teóricos especializados.

Es acertado que en los textos de Hawkins y otros divulgadores científicos que son considerados como pilares de la divulgación científica en la comunidad general, se caracterizan por tener un lenguaje sencillo, un uso de imágenes pertinentes para simplificar explicaciones, una disminución de contenido matemático especializado (o bien, un uso simplificado del mismo) y también de que abundan de elementos que son funcionales para una expansión de las investigación así como para la realización transmediática.

\section{Una historia de nativos, divulgadores, canales y transmedia}

Como fue establecido anteriormente, se realizó un trabajo de investigación de campo cuyo objetivo era el diseño de una propuesta de canales complementarios de comunicación científica. Esto, basado en el consumo informativo de una muestra compuesta por nativos digitales y divulgadores, mismo que fue realizado entre los años 2017 y 2018 en Puebla, México.

Dicha investigación, llevó una fuerte revisión de bibliografía para la creación de un marco teórico, contextual y conceptual. Una vez realizada la investigación que soportaría las necesidades, se plantearon las estrategias metodológicas para la realización de un estudio de dos muestras. Esto con 
el fin de poder identificar por una parte la perspectiva de los nativos digitales ante la divulgación científica y por otra parte la perspectiva de los divulgadores científicos. Ante el reto, se buscó contrastar la información de primera y segunda fuente para poder identificar áreas de oportunidad en una propuesta de modelo de canal complementario.

El tipo de investigación fue de tipo mixto (cualitativo y cuantitativo) de estatus dominante: dándole mucho mayor peso a la investigación cualitativa. Se realizó un estudio explicativo de tipo observacional: es decir, que el investigador tuvo la oportunidad de recopilar diferentes datos para poder hacer un análisis en cuanto a la relación entre las causas y las consecuencias. Por último, se tomó en cuenta la revisión de estudios de caso y de especialistas en la materia para poder obtener información y generar un análisis integral.

Dentro de los primeros resultados arrojados, para la mayoría de los nativos digitales encuestados, el interés y la relación con la divulgación científica es baja. Esto debido a un sentimiento de tedio uniforme sobre la percepción que genera la lectura o el acercamiento a temas de ciencia. Para este grupo de estudio, en su mayoría, la divulgación científica resulta aburrida o difícil de entender. Aquellos que tienen un acercamiento con artículos científicos predisponen una percepción negativa sobre ello, debido a que se les ha obligado a la lectura en sus cursos universitarios o bien la proximidad con el área de las ciencias representa un esfuerzo involuntario.

La falta de tiempo, comprensión, desinterés en la lectura o en los temas de ciencia, son los principales ejes que deben ser atacados para que la divulgación científica pueda permear el interés de este grupo mayoritario que bien, no demuestra atención o esfuerzo por generar un vínculo con la faena de la divulgación científica tanto como usuario final así como productor de contenido.

Uno de los problemas identificados con los nativos digitales es en el tópico de la escasez de lectura y acercamiento a divulgaciones científicas. Uno de los principios que buscan justificar este desinterés, está supuestamente basado en los programas académicos de las universidades que, más que promover, imponen la lectura de los mismos textos que forman parte del presente análisis por falta de canales complementarios para llegar a nuevos públicos.
Como resultado en los nativos digitales que se alejan del quehacer científico, se habla del desencadenamiento de respuestas negativas como el desinterés científico, la apatía académica, la percepción sobre las divulgaciones y los divulgadores científicos, entre otros aspectos importantes. Uno de los principales riesgos con este grupo, es que si las corrientes de enseñanza en las universidades y el impacto negativo de los divulgadores en los nativos digitales puede crear un alejamiento mayor al grado que la propuesta de canales complementarios sea difícil de instalar en la sociedad.

Por otra parte, aquellos nativos digitales que sí consumen productos de divulgación científica, encuentran las divulgaciones científicas como un medio para la construcción de su persona a nivel profesional así como académico. De este grupo, encontramos oportunidades y variantes listas para ser explotadas en un proceso de experimentación y asociación.

Conforme a la muestra de divulgadores científicos, los resultados evidencian lo siguiente: los divulgadores científicos presentan un desinterés supuestamente fundamentado en su contexto tecnológico. El divulgador mismo no se enfrenta directamente a los retos mediáticos, sino se confía de sus conocimientos básicos para poder comunicarse y utilizar los medios de comunicación tradicionales ya existentes para beneficiar su tarea de divulgación.

El divulgador científico cree que los canales de comunicación complementarios sí son utilizados: esto al afirmar que la mejor manera de compartir información es utilizando herramientas como los hipervínculos a páginas de diseño web poco atractivo, o la gestión de cápsulas informativas en sitios de difícil acceso.

Los divulgadores sostienen comprender el cómo se utiliza el internet, aunque sólo hablan de publicar en redes sociales como una herramienta funcional y en la que pueden llegar a confiar de vez en cuando. Su conocimiento sobre aspectos básicos como la interacción o el engagement, podría formar parte del fenómeno de desinterés en el uso de nuevas herramientas digitales en apoyo a su trabajo.

Retomando el tema de los canales complementarios que promueven las investigaciones y las publicaciones de los divulgadores, estos últimos afirman que en la mayoría de los casos, no investigan qué medios son los indicados para divulgar, ya que sostienen que no tienen tiempo para ello, 
por lo que prefieren los ya conocidos (publicación en revistas especializadas para la academia). Podríamos inferir que el proceso de divulgación se puede ver empañado por cuestiones de comodidad y menor esfuerzo.

\section{Conclusiones}

Nos encontramos ante un grupo de presuntos culpables. ¿Quiénes son los responsables de dicha crisis de la divulgación científica? ¿Son por una parte, los divulgadores científicos ante el desinterés colectivo en adaptarse a nuevas formas de comunicación y no tomar en cuenta a nuevos públicos para un proyecto editorial mucho más ad hoc? o ¿son los nativos digitales quienes bajo un hipotético empoderamiento social solicitan urgentemente que los medios y los contenidos se adapten a ellos; y no ellos a los medios actuales? Sin olvidar que este grupo también goza de una supuesta crisis generacional que busca justificar el desinterés en temas como la ciencia y la tecnología fuera de las obligaciones académicas.

Por otra parte, ¿son acaso los medios de comunicación y su constante cambio los que no permiten la creación y fortalecimiento de un sistema de comunicación funcional e integral? O es tal vez ila falta de empoderamiento, sensibilización y concientización de la sociedad en general la que no exige de manera global los análisis y las soluciones necesarias a sus gobiernos, sociedades del conocimiento, así como futuras y viejas generaciones?

En realidad, la culpa es general. Por lo tanto, la responsabilidad de una solución es de corte, colectivo y con función hacia un mejor mañana. la divulgación científica es un proceso que nos involucra a todos, sin importar el papel que optemos tener: el de usuario pasivo o activo, el de creador de contenido, el de expansor de investigación, el de auditor o el de consultor. La divulgación científica, su adaptación y su futuro, es un tema que aborda a las personas, sus gobiernos y a quienes nos prosiguen.

Para que la comunidad de divulgación científica pueda fortalecer su tarea en el país, debe y está obligada a contar con las herramientas necesarias para hacerlo. Las nuevas narrativas son herramientas de participación y afiliación a las nuevas formas de consumo de información. Hoy en día, el transmitir historias, basa mucho su éxito en la forma en que estas son contadas. Aprender a utilizar las narrativas de forma útil y eficaz, puede generar en las ciencias nuevos puntos de apoyo para comunicar y llegar a nuevos públicos. La comunicación jamás debe ser desvalorizada. Si la comunicación es buena, habrá que prestar atención. Si la comunicación es mala, habrá que destinar mucho mayor interés.

\section{Referencias $^{3}$}

Calvo, M. (2005). Ciencia y periodismo científico en Iberoamérica. Recuperado el 5 de febrero de 2017 de http://www.lainsignia.org/2005/abril/dial_005.htm

COSNET. (1985). Experiencias de la divulgación de tecnología y ciencia en México. México: SEP/COSNET

Estrada, Luis. (1992). La divulgación de la ciencia. Recuperado el 6 de febrero de 2017 de http://www. revistaciencias.unam.mx/pt/175-revistas/revista-cienci as-27/1620-la-divulgaci\%C3\%B3n-de-la-ciencia.html

Estrada, L. (2014). La comunicación de la ciencia. Recuperado el 21 de enero de 2017 http://www.revi sta.unam.mx/vol.15/num3/art18/index.html

Hatch, R. (s.f.). Histoire de l'Académie Royale des Sciences. Recuperado de http://users.clas.ufl.edu/ufhatch/pages/// 02-TeachingResources/ClioElectric/1-Electronic\%20T exts/Hatch-Acad\%20des\%20Science\%20-\%20Prefac e\%20-\%20Histoire\%201699\%20-\%20Eng.pdf

Hawkins, S. (1988). A brief history of time. Inglaterra: Bantam Books.

Howe, N., Strauss, W. (1991). Generations. Recuperado en agosto de 2017 de https://books.google.com.mx/books/ about/Generations.html?id=FTGY-uoCCCoC\&redir_e $\mathrm{sc}=\mathrm{y}$

Ibarra Arias, José Juan Antonio, \& Villagrán Guerrero, Alejandra Elizabeth (2013). La investigación en las universidades privadas: ¿un lujo o una necesidad?. Universidades, (55), undefined-undefined. Recuperado en octubre 2017 de https://www.redalyc.org/articulo.o a?id=373/37331244010

Jenkins, H. (2011). Three Reasons Why Pottermore Matters. . Recuperado en marzo 2018 de http://henryjenkins.org/b log/2011/06/three_reasons_why_pottermore_m.html

Negrete A. 2008. La Comunicación Científica a Través de Formas Narrativas. México: Libro en Coedición DGDC-CEIICH (UNAM)

Prensky, M. (2001). Nativos e Inmigrantes digitales. Recuperado de https://ies28-sfe.infd.edu.ar/aula/archivo s/repositorio/0/88/Prensky_Nativos_e_inmigrantes.pdf

\footnotetext{
${ }^{3}$ Para el trabajo Proyecto Integrador: Canales complementarios de comunicación científica basado en el consumo informativo de los Nativos Digitales en Puebla, 2017 - 2018. fueron utilizadas más de 250 fuentes de información. Favor de contactar al autor en caso de solicitar la compartición del trabajo original.
} 
Royal Society Org. (s.f.). History. Recuperado de https://roya lsociety.org/ /media/Royal_Society_Content/about-us/ history/Charter1_English.pdf?la=en-GB

Sanders, M (2009). "STEM, STEM Education, STEMmania". The Technology Teacher. International Technology Education Association. EUA

Scolari, C. (2014). Narrativas transmedia: nuevas formas de comunicar en la era digital. Recuperado de octubre de 2017 https://www.accioncultural.es/media/Default $\% 20$ Files/activ/2014/Adj/Anuario_ACE_2014/6Transmedi a_CScolari.pdf

UNAM. (2013) Fuga de cerebros, la diáspora del conocimiento. Recuperado en octubre 2017 de http: //ciencia.unam.mx/leer/150/Fuga_de_cerebros_la_dia spora_del_conocimiento

UNESCO. (1999). Declaración de la Ciencia sobre la ciencia y el uso del saber científico. Recuperado octubre de 2018 de http://www.unesco.org/science/wcs/esp/declaracion _s.htm 